\title{
Response to 'Comment on: ophthalmology specialist trainee survey in the United Kingdom'
}

\author{
William H. Dean ${ }^{1,2} \cdot$ Susannah Grant ${ }^{3,4} \cdot \operatorname{Jim}$ McHugh $^{5} \cdot$ Oliver Bowes $^{6} \cdot$ Fiona Spencer $^{3,7}$
}

Received: 17 October 2019 / Accepted: 17 October 2019 / Published online: 11 December 2019

(c) The Author(s), under exclusive licence to The Royal College of Ophthalmologists 2019

\section{To the Editor:}

We agree with the points raised by Dr Nairn et al. and that running a regular simulation-based surgical skill (“dry-lab”) session would enable safe and efficient acquisition of competence. This is why, through the Training Committee of the Royal College of Ophthalmologists, the Simulation lead and Surgical Skills lead have set all regions the target of ensuring simulation training in cataract complications, namely vitreous loss, is undertaken at least annually in each region for all trainees.

The Simulated Ocular Surgery Trials are two separate educational-intervention randomised controlled trials. The first of these, the OLIMPICS (ophthalmic learning and improvement initiative in cataract surgery) trial will be published early next year. These are the first ever prospective RCTs evaluating the utility of intense simulationbased surgical education versus conventional training alone in cataract and glaucoma surgery.

\section{Compliance with ethical standards}

Conflict of interest The authors declare that they have no conflict of interest.

Publisher's note Springer Nature remains neutral with regard to jurisdictional claims in published maps and institutional affiliations.

William H. Dean

will.dean@1shtm.ac.uk

1 International Centre for Eye Health, London School of Hygiene and Topical Medicine, London, UK

2 Community Eye Health Institute, University of Cape Town, Groote Schuur Hospital, Cape Town, South Africa

3 The Royal College of Ophthalmologists, London, UK

4 The Royal College of Emergency Medicine, London, UK

5 Department of Neuro-Ophthalmology, National Hospital for Neurology and Neurosurgery, University College London Hospital NHS Trust, London, UK

6 Department of Ophthalmology, Cambridge University Hospitals NHS Foundation Trust, Cambridge, UK

7 Manchester University NHS Foundation Trust, Manchester, UK 